\title{
Percepções de estudantes de Odontologia sobre a contribuição do preceptor
}

\author{
Ana Lucia Herdy Teixeira*; Jaqueline Vilela Bulgareli**; Flávia Martão Flório***; Luciane Zanin*** \\ * Mestre em Saúde Coletiva, Faculdade São Leopoldo Mandic \\ ** Doutora em Saúde Coletiva, Faculdade de Odontologia de \\ Piracicaba \\ *** Doutora em Cariologia, Docente, Faculdade São Leopoldo \\ Mandic
}

Recebido em 13/04/2018. Aprovado em 23/02/2019.

\begin{abstract}
RESUMO
O objetivo deste estudo foi identificar a percepção de discentes de Odontologia acerca da contribuição dos preceptores no seu processo de formação acadêmica. Este estudo qualitativo abordou 69 estudantes de três Instituições de Ensino Superior públicas e privadas do município do Rio de Janeiro. Os voluntários responderam sobre suas percepções a respeito da contribuição do preceptor no processo formativo. As respostas foram avaliadas pela análise de conteúdo de Bardin e classificadas em 3 categorias temáticas: "A experiência/conhecimento clínico necessário no ensino", "O entendimento do processo de trabalho na Atenção Primária" e "A capacitação profissional". Concluise que, sob a perspectiva do discente, o preceptor é considerado um profissional com perfil necessário para atender às suas demandas acadêmicas, já que possui o conhecimento clínico necessário no ensino e conhece o processo de trabalho no serviço público. A falta de capacitação profissional pode influenciar o processo de aprendizado.
\end{abstract}

Descritores: Preceptoria. Capacitação Profissional. Sistema Único de Saúde. Educação Superior.

\section{INTRODUÇÃO}

O Pró-PET saúde vem se estabelecendo como importante instrumento para adequação às Diretrizes Curriculares Nacionais (DCN) para os cursos da área da saúde, incorporando o discente a cenários de prática do Sistema Único de Saúde (SUS), visando à aproximação entre Instituições de Ensino Superior públicas e privadas e os serviços 
públicos de saúde. Contribui com um ensino compatível com as necessidades de saúde da população a partir da produção de conhecimento que faça sentido no modo de profissionais e estudantes pensarem e agirem em saúde para a prestação de um serviço de qualidade à população brasileira ${ }^{1}$.

A partir da integração ensino-serviço preconizada pelos Ministérios da Saúde e Educação surgiu uma nova responsabilidade para os profissionais vinculados ao serviço público de saúde que, nos dias atuais, vêm assumindo tarefas ligadas ao processo de ensino-aprendizado. Os estudantes acompanham o trabalho, aplicam conhecimentos teórico-práticos e esclarecem dúvidas durante o período do estágio ${ }^{2}$.

Assim, os preceptores vêm sendo reconhecidos como mediadores da integração dos estudantes à rotina de trabalho no serviço público ${ }^{3}$, contribuindo, com sua experiência de trabalho nos serviços, para o melhor entendimento do estagiário sobre o funcionamento do setor público ${ }^{4}$, das necessidades de saúde da população ${ }^{5}$, despertando o interesse pelo trabalho no serviço público ${ }^{6}$. Esta atuação do preceptor está prevista pelas DCN para os cursos de Odontologia, que em seu $5^{\circ}$ artigo, inciso VI, ditam que os profissionais devem ter responsabilidade e compromisso com o treinamento das futuras gerações de profissionais ${ }^{7}$.

Estudos demonstram que o preceptor é reconhecido como parte importante do processo de formação de discentes ${ }^{3-6}$, porém é necessária uma reflexão sobre sua qualificação para as mudanças das práticas de saúde exigidas atualmente ${ }^{4}$, particularmente quanto à capacitação pedagógica para a preceptoria ${ }^{5,9,10}$, compreensão de seu verdadeiro papel no processo de formação discente $^{11}$ e como conciliar a preceptoria com a carga de trabalho clínica e/ou administrativa ${ }^{12}$.

Diante do exposto, este estudo teve como objetivo conhecer a percepção dos discentes de Odontologia acerca da contribuição dos preceptores no seu processo de formação acadêmica.

\section{METODOLOGIA}

O presente estudo de abordagem qualitativa foi conduzido conforme determinado pela Resolução 466 de 12/12/2012 do Conselho Nacional de Saúde do Ministério da Saúde e aprovado no Comitê de Ética em Pesquisa (CAAE: 38109914.6.0000.5374).

A população estudada foi composta por discentes de Odontologia do município do Rio de Janeiro que concluíram o Estágio Supervisionado na Atenção Primária à Saúde, nas modalidades Unidades Básicas de Saúde e/ou Unidades de Saúde da Família e que foram supervisionados/ orientados por preceptores do serviço público.

Assim, foram convidados a participar da pesquisa 73 estudantes de três universidades, denominadas " $X$ ", "Y" e "Z", sendo duas públicas e uma privada, sendo excluídos da pesquisa estudantes que estavam ausentes nos dias agendados para as entrevistas.

A questão norteadora do roteiro teve como base a percepção dos discentes em relação à contribuição do preceptor na sua formação acadêmica. Os questionários foram lidos e as respostas foram avaliadas a partir da análise de conteúdo $^{13}$, a qual possui um significado especial no campo das investigações sociais, representando uma abordagem metodológica com características e possibilidades próprias para atingir níveis de compreensão mais aprofundados, impossíveis de serem contabilizados matematicamente ${ }^{14}$.

As respostas transcritas foram lidas pelo pesquisador e seguiu-se a categorização dos depoimentos dos discentes.

\section{RESULTADOS E DISCUSSÃO}

Fizeram parte do estudo 69 discentes ( 24 da universidade " $\mathrm{X}$ ", 18 da "Y" e 27 da "Z"). O conjunto de respostas gerou 5 ideias centrais sendo 
3 discutidas neste estudo, a saber: "A experiência/conhecimento clínico necessário no ensino"; "O entendimento do processo de trabalho na Atenção Primária” e “A capacitação profissional".

\section{A experiência/conhecimento clínico necessário no ensino}

Os estudantes perceberam os preceptores como profissionais capacitados para atender suas demandas, já que apontaram que possuem experiência e/ou conhecimento clínico para exercer as atividades de preceptoria, dando bastante ênfase à essa questão em suas falas:

"Todos os profissionais que me supervisionaram em minha graduação foram de alta capacidade, habilidade prática e conhecimento cientifico" (4X).

"Tem conhecimento teórico e prático para exercer o cargo" (8Y).

"Além de formação, conhecimentos técnicos, são profissionais que tem muita experiência em virtude da demanda de atendimentos" (14Z).

A aquisição da experiência clínica constitui uma parte importante da graduação e da pósgraduação na área da saúde, e o preceptor é o profissional que tem como função primordial auxiliar no desenvolvimento das habilidades clínicas do estudante. Assim, ter conhecimento e habilidade para desempenhar procedimentos clínicos são requisitos imprescindíveis na preceptoria de estudantes de graduação, pois durante o estágio supervisionado as atividades clínicas ocupam um espaço privilegiado na rotina dos discentes ${ }^{15}$.

Relatos na literatura demostram que algumas vezes preceptores dominam os saberes profissionais, mas não os saberes pedagógicos ${ }^{9}$. Os desafios da ação pedagógica na preceptoria são grandes e perpassam competências distintas daquelas exigidas pela clínica ${ }^{16}$, o que significa dizer que nem sempre um bom clínico possui habilidades e competências para a preceptoria.

Embora nesse estudo não tenha ocorrido relatos de necessidade de capacitação pedagógica do preceptor para exercer as atividades de ensino, é considerado indispensável por diversos autores $^{5,9,10}$.

Muitas vezes os profissionais que atuam como preceptores acabam reproduzindo um ensino puramente técnico, baseado no passo a passo clínico, entretanto a sua função como preceptor deve ir além do repasse da prática clínica diária no serviço de saúde ${ }^{17}$. Assim, deve ter um conhecimento ampliado dos aspectos que afetam a saúde de comunidades e populações para proporcionar uma atenção integral e contínua. Sendo assim, os estudantes devem ser incluídos nas rotinas dos serviços de saúde para que possam produzir um conhecimento além das práticas clínicas.

\section{O entendimento do processo de trabalho na Atenção Primária}

Outra justificativa dos discentes para considerar o preceptor capacitado para receber estudantes nos campos de prática foi considerar que o mesmo possui conhecimento do processo de trabalho na Atenção Básica.

"É uma pessoa que conhece a rotina acadêmica de consultório e dos pacientes, portanto, consegue fazer um bom planejamento para as atividades de graduação” (11Y).

"Eles já estão acostumados e conseguem levar os alunos muito bem e nos deixam bem confortáveis" (17Z).

A vivência diária do preceptor nos serviços de saúde confere característica de apropriação do processo de trabalho e da equipe. Assim, o trabalho em saúde deve ser entendido como coletivo, que apesar das especificidades de conhecimentos e de práticas profissionais faz parte de um conjunto que 
resulta na assistência à saúde de seres humanos. Sua compreensão torna-se imprescindível para a prestação de uma assistência de qualidade, desenvolvida por seres humanos que veem o produto de seu trabalho valorizado socialmente ${ }^{4}$.

Além disso, o processo de trabalho em saúde deve contemplar um conjunto de ações coordenadas e orientadas, de caráter individual e coletivo, desde a promoção de saúde à reabilitação, a fim de modificar a situação de saúde da população, seus determinantes e condicionantes ${ }^{1}$.

Assim, há a necessidade de o preceptor guiar e estar engajado nessa perspectiva, pois ensinar os conteúdos específicos ou apenas clinicar exige momentos de reflexão e crítica sobre o processo de trabalho em saúde, o que vem sendo aos poucos percebido e valorizado pelos preceptores em seus momentos pedagógicos ${ }^{7}$.

Dessa forma, os estudantes que possuem experiências prévias de atuação em campos de estágio no SUS, guiados por trabalhadores de saúde preparados e que estejam vivenciando situações reais ligadas à rotina de trabalho, às necessidades dos serviços, ao trabalho interprofisssional em equipe, ao funcionamento e gestão do serviço público, estarão preparados para atuar no futuro mercado de trabalho, oferecendo um serviço de qualidade para a população.

\section{A capacitação profissional}

Os profissionais de hoje são frutos do modelo de formação enquanto estudantes ${ }^{11,18,19} \mathrm{e}$, muitas vezes, não possuem treinamento ou perfil adequado para atuar como preceptor e acabam desestimulando os estagiários em sua prática, pois esses percebem quando o profissional está desmotivado, desatualizado ou sobrecarregado na sua função ${ }^{12,20}$.

Nesse estudo, muitos participantes observaram o preceptor como um profissional capacitado/treinado para exercer as atividades da preceptoria, embora outros discutam que a capacitação do profissional está ligada à questão da atualização.

"Pois ele é uma pessoa treinada para auxiliar os alunos" (6X).

"Todos os preceptores que tive a oportunidade de conhecer, eram ótimos profissionais, visivelmente capacitados as suas funções” (2Z).

“... São profissionais capacitados que estão se atualizando" (7X).

"Durante o meu estágio supervisionado o preceptor mostrou-se capacitado para as diversas situações. No entanto é necessário promover atitudes para manter esse preceptor sempre atualizado" (12X).

A ausência da educação continuada do preceptor aparece como uma questão que pode vir a interferir negativamente no aprendizado do estagiário, gerando uma dicotomia entre o ensino praticado nas universidades e o nos campos de estágio.

“... Único problema que percebo é a atualização em algumas áreas, muitas vezes, o preceptor ainda não teve contato com as novas técnicas que aprendemos na faculdade, por isso a educação permanente em saúde é importante" (17X).

"A falta de atualização do meu preceptor me deixou muito confusa em certas situações" (23X).

A realização do estágio com o suporte do preceptor contribui também para ampliar a experiência e vivência do estudante, oportunizando a aquisição de novos conhecimentos que lhe servirão para sua futura profissão, embora uma das sugestões tenha sido a capacitação quanto ao conhecimento científico ${ }^{7}$ como percebido nessa pesquisa.

As DCN para o curso de odontologia, em seu artigo 4, inciso VI, citam a Educação Permanente, tanto na formação quanto na prática profissional. Assim, é fundamental aprender a aprender, ter responsabilidade e compromisso com a própria 
educação e no treinamento das futuras gerações de profissionais, proporcionando as condições necessárias para que haja benefício mútuo entre os profissionais do serviço e estudantes ${ }^{7}$. Torna-se responsabilidade do preceptor a busca por constante aperfeiçoamento, mas é necessário que as três esferas do governo e as Instituições de Ensino Superior incentivem e promovam oportunidades de educação continuada para esses profissionais.

Por conseguinte, é importante relatar que os diversos cenários de práticas já se concretizam como espaços para a construção de novos saberes, atitudes e valores que serão responsáveis por auxiliar no processo de qualificação profisssional em saúde, a partir da reflexão da própria prática diária $^{10}$.

A capacitação/atualização profissional também acontece a partir do trabalho com os estudantes, despertando no preceptor a necessidade de qualificação permanente para o do trabalho em saúde ${ }^{5,20}$, já que a inserção de estudantes nas atividades do estágio estimula que os profissionais busquem o conhecimento teórico e acadêmico para conseguir dialogar com a universidade e não se sentirem desatualizados perante os estagiários ${ }^{12,21}$

A presença de discentes no serviço é um estímulo para a atualização, além de tornar as atividades mais dinâmicas, prazerosas e humanas, proporcionando crescimento pessoal e profissional dos envolvidos. A convivência de estudantes e docentes permite maior proximidade com a universidade e gera nos preceptores um desejo maior de buscar o aprimoramento necessário ${ }^{5}$.

O processo educativo atual não está mais centrado em uma única figura detentora do saber e sim constitui-se em um processo de troca entre os atores envolvidos, já que "quem ensina aprende ao ensinar e quem aprende ensina ao aprender" ${ }^{22}$, fato percebido na fala dos discentes avaliados nesse estudo.

"Eles possuem bastante conhecimento tanto teórico quanto prático e gostam de trabalhar com pessoas mais atualizadas então ele além de aprender eles ensinam" (24X).

Apesar de vários autores demostrarem a necessidade de capacitação e/ou atualização do preceptor $^{6,10,15,23}$, outros mostram que a educação permanente e a qualificação em serviço de recursos humanos para a saúde vêm ocorrendo a partir de projetos como o Pró-PET-Saúde ${ }^{9,24}$.

\section{CONCLUSÃO}

Esse estudo evidenciou, sob perspectiva discente, que os preceptores são capacitados para atender às demandas dos estudantes de graduação durante o período do Estágio Supervisionado, contribuindo com o processo de formação discente, pois possuem conhecimento clínico, do processo de trabalho no serviço público, e estão aptos a guiar o discente durante as atividades, com perfil necessário para exercer a preceptoria. No entanto, os entrevistados sentiram falta da educação continuada dos preceptores.

\section{ABSTRACT \\ Perceptions of Dentistry students regarding the contribution of preceptors}

The aim of the present study was to identify the perception of students of the Dentistry regarding the contribution of preceptors in their process of academic training. This qualitative study involved 69 volunteer students from three public and private higher education institutions in the city of Rio de Janeiro. The volunteers described their perceptions regarding the contribution of the preceptor in their training process. The answers were evaluated through Bardin's content analysis and classified into 3 thematic categories: "The clinical knowledge/experience required for teaching"; "The understanding of the process of working in Primary Care" and "Professional training". It was concluded that, from the 
perspective of the students, the preceptor is considered to have the necessary characteristics to meet their academic demands needs, as they have the necessary clinical knowledge and teaching knowledge and understand the working process in public service. A lack of professional training can influence the learning process.

Descriptors: Preceptorship. Professional Training. Unified Health System. Education Higher.

\section{REFERÊNCIAS}

1. Brasil. Ministério da Saúde. Portaria $N^{o}$ 2.805, de 6 de dezembro de 2012. Define os valores para a implementação do Programa Nacional de Reorientação da Formação Profissional em Saúde - Pró-Saúde, a ser repassado aos respectivos Fundos Estaduais e Municipais de Saúde em parcela única. Brasília: Ministério da Saúde, 2012.

2. Gonçalves CM, Santos KT, Carvalho RB. O PET-Saúde como instrumento de reorientação do ensino em Odontologia: a experiência da Universidade Federal do Espírito Santo. Rev ABENO. 2011;11(2):273.

3. Luz GW, Toassi RFC. Percepções sobre o preceptor cirurgião-dentista da Atenção Primária à Saúde no ensino da Odontologia. Rev ABENO. 2016;16(1):2-12.

4. Souza MIDCD, Maia KD, Jorge RR, Berlink T, Ramos MEB. Análise discente da contribuição do preceptor e do estágio na formação do aluno de graduação da FO UERJ. Ver ABENO. 2011;11(2):57-62.

5. Lima PAB, Rozendo CA. Desafios e possibilidades no exercício da preceptoria do Pró-PET-Saúde. Interface. 2015;19(Suppl 1):779-91.

6. Bulgarelli AF, Souza KR, Baumgarten A, Souza JM, Rosing CK. Formação em saúde com vivência no Sistema Único de Saúde
(SUS): percepções de estudantes do curso de Odontologia da Universidade Federal do Rio Grande do Sul (UFRGS), Brasil. Interface. 2014;18(49):351-62.

7. Conselho Nacional de Educação. Câmara de Educação Superior. Institui Diretrizes Curriculares Nacionais do Curso de Graduação em Odontologia. Resolução CNE/CES 3/2002. Diário Oficial da União, Brasília, 4 de março de 2002. Seção 1, p. 10.

8. Batista KBC, Gonçalves OSJ. Formação dos profissionais de saúde para o SUS: significado e cuidado. Saúde Soc. 2011;20(4):884-99.

9. Pinto ACM, Oliveira IV, Santos ALS, Silva LES, Izidoro GSL, Mendonça RD, et al. Percepção dos alunos de uma universidade pública sobre o Programa de Educação pelo Trabalho para a Saúde. Ciênc Saúde Coletiva. 2013;18(8):2201-10.

10. Autonomo FROM, Hortale VA, Santos GB, Botti SHO. A Preceptoria na formação médica e multiprofissional com ênfase na Atenção Primária - análise das publicações brasileiras. Rev Bras Educ Méd. 2015;39(2):316-27.

11. Monteiro DM, Leher EM, Ribeiro VMB. A formação pedagógica dos profissionais da Área da Saúde. Formação pedagógica de preceptores do ensino em saúde. Juiz de Fora: Editora UFJF; 2011. p. 13-22.

12. Vasconcelos ACF, Stedefeldt E, Frutuoso MFP. Uma experiência de integração ensinoserviço e a mudança de práticas profissionais: com a palavra, os profissionais de saúde. Interface Comun Saúde Educ. 2016 mar;20(56):147-58.

13. Bardin L. Análise de Conteúdo. Portugal: Edições 70; 2009.

14. Moraes R. Análise de conteúdo. Rev Educ. 1999;22(37):7-32.

15. Rocha PF. O preceptor cirurgião-dentista da 
atenção primária à saúde na formação em odontologia: compreensão do papel e análise das características para a preceptoria [dissertação]. Porto Alegre: Faculdade de Medicina da Universidade Federal do Rio Grande do Sul; 2014.

16. Rocha HCR, Ribeiro VB. Curso de formação pedagógica ara preceptores do internato médico. Rev Bras Educ Méd. 2012;36(3):343-50.

17. Oliveira ET, Vasconcelos M, Rodarte RS, Esteves RZ. Reflexões sobre a prática pedagógica do Cirurgiões-Dentistas preceptores de estágio. CIAIQ. 2017; 2:25969.

18. Marins JJN. Formação de preceptores dos para a Área da Saúde. In: Brant V (Org.). Formação pedagógica de preceptores do ensino em saúde. Juiz de Fora: Editora UFJF; 2011. p. 47-52.

19. Mohr A. A Formação Pedagógica dos Profissionais da Área da Saúde. In: Brant V (Org.). Formação pedagógica de preceptores do ensino em saúde. Juiz de Fora: Editora UFJF; 2011. p. 53-66.
20. Forte FDS, Pessoa TRRF, Freitas CHSM, Pereira CAL, Carvalho Junior PM. Reorientação na formação de cirurgiõesdentistas: o olhar dos preceptores sobre estágios supervisionados no Sistema Único de Saúde (SUS). Interface Comun Saúde Educ. 2015;19 (Supl. 1):831-43.

21. Pinto TR, Cyrino EG. Com a palavra, o trabalhador da Atenção Primária à Saúde: potencialidades e desafios nas práticas educacionais. Interface Comun Saúde Educ. 2015;19 (Supl 1):765-77.

22. Freire P. Pedagogia da Autonomia. São Paulo, Paz e Terra; 1997.

23. Barreto VHL, Marco MAD. Visão de Preceptores sobre o processo de ensinoaprendizagem no internato. Rev Bras Educ Méd. 2014;38(1):94-102.

24. Moura TP, Costa RCM, Damasceno TC, Nuto SAS, Pequeno LL. Percepção da inserção de alunos na atenção primária: visão dos gestores e profissionais da Estratégia Saúde da Família. Rev ABENO. 2010;10(2):46-52.

\section{Correspondência para:}

Luciane Zanin

e-mail: zanninsouza@yahoo.com.br

Rua Prebítero Francisco Rocha de Oliveria , 47

Residencial Reserva da Serra

13.212-154 Jundiai/SP 